\title{
Medullary metastasis of a malignant peripheral nerve sheath tumor: A case report
}

\author{
TOMOHITO HAGI $^{1}$, TOMOKI NAKAMURA ${ }^{1}$, AYUMU YOKOJI ${ }^{2}$, AKIHIKO MATSUMINE $^{1}$ and AKIHIRO SUDO ${ }^{1}$ \\ ${ }^{1}$ Department of Orthopedic Surgery, Mie University Graduate School of Medicine; ${ }^{2}$ Department of Anesthesiology, \\ Mie University Hospital, Tsu, Mie 514-8507, Japan
}

Received March 19, 2015; Accepted April 1, 2016

DOI: $10.3892 / \mathrm{ol} .2016 .4872$

\begin{abstract}
The present study reports a case of medullary metastasis without lung metastasis that occurred as a result of a malignant peripheral nerve sheath tumor (MPNST). An 81-year-old woman presented with a MPNST in the left brachial plexus, arising from the cervical nerve root. The patient underwent carbon ion radiotherapy; however, tumor recurrence was identified in the left shoulder. Subsequently, the patient underwent wide excision. Three weeks subsequent to surgery, imbalance and dysarthria developed suddenly. Dysphagia emerged and left upper limb pain disappeared on the day after symptom development. Magnetic resonance imaging (MRI) revealed that this was due to metastasis to the medulla. Five days subsequent to the onset of dysarthria, the patient succumbed due to respiratory failure. To the best of our knowledge, no previous cases of medullary metastasis arising from a MPNST in the absence of lung metastasis have been reported. MRI is a useful examination tool for the identification of brain metastases; however, the high cost of MRI as a routine examination must be considered due to the rarity of brain metastases. Therefore, methods to detect brain metastasis warrant further investigation.
\end{abstract}

\section{Introduction}

Malignant peripheral nerve sheath tumors (MPNSTs) are highly malignant sarcomas derived from the neural crest, and account for $5-10 \%$ of all soft tissue sarcomas (1). MPNSTs exhibit a high incidence of local recurrence and distant metastases. Metastases are most frequently identified in the lung, followed by the bone and pleura (2). Currently, there are no recommended adjuvant treatments for MPNST as there is for other soft tissue sarcomas. The European Society for Medical Oncology guidelines state

Correspondence to: Dr Tomoki Nakamura, Department of Orthopedic Surgery, Mie University Graduate School of Medicine, 2-174 Edobashi, Tsu, Mie 514-8507, Japan

E-mail: tomoki66@clin.medic.mie-u.ac.jp

Key words: brainstem metastasis, malignant peripheral nerve sheath tumor that surgery remains to be the optimal treatment, with the aim of achieving clear surgical margins. Despite multimodal treatment, the prognosis of MPNST is poor with 5-year survival rates between 23-69\% (3). Brain metastasis from soft tissue sarcomas, including MPNST, is rare with a reported prevalence of $1-6 \%$ of all soft tissue sarcomas $(1,4,5)$. In particular, brain metastasis arising from soft tissue sarcomas, in the absence of lung metastasis, is considered a relatively rare event $(1,4,5)$. Furthermore, to the best of our knowledge, no cases of medullary metastasis without preceding lung metastasis have been reported in the literature to date. The current study presents the case of an 81-year-old woman with medullary metastasis arising from a MPNST of the brachial plexus, who experienced loss of medullary function.

\section{Case report}

In July 2012, an 81-year-old woman presented to Shingu Municipal Medical Center (Shingu, Japan) with numbness in the upper left arm. At this hospital, the patient was diagnosed with unknown neuritis and thus received no treatment. A total of 6 months later in January 2013, the patient was referred to Mie University Hospital (Tsu, Japan) due to paralysis of the left arm, which was suspected to be a disorder of the brachial plexus. Clinically, no neck or shoulder masses were evident during physical examination. The patient was unable to contract any muscles in the left upper extremity and experienced severe pain in the upper left limb, which could not be relieved by major analgesics (including tramadol hydrochloride $112.5 \mathrm{mg} / \mathrm{day}$ and acetaminophen $975 \mathrm{mg} /$ day). The patient was administered oxycodone (10 mg/day) and pregabalin (125 mg/day), which successfully alleviated the pain. A fluorodeoxyglucose positron emission tomography-computed tomography (CT) scan (Discovery PET/CT 690; GE Healthcare Bio-Sciences, Pittsburgh, PA, USA) revealed increased tracer uptake in the left brachial plexus (Fig. 1A) arising from the cervical nerve root (Fig. 1B), which indicated the presence of a tumor. Magnetic resonance imaging (MRI; Ingenia 1.5T; Philips Healthcare, DA Best, The Netherlands) identified a soft tissue mass along the nerve at the left brachial plexus, which exhibited low signal intensity on T1-weighted images and high signal intensity on T2-weighted images.

A CT-guided needle biopsy was subsequently performed. Tissues were cut into $3.0 \mu \mathrm{m}$ sections and stained with 
A

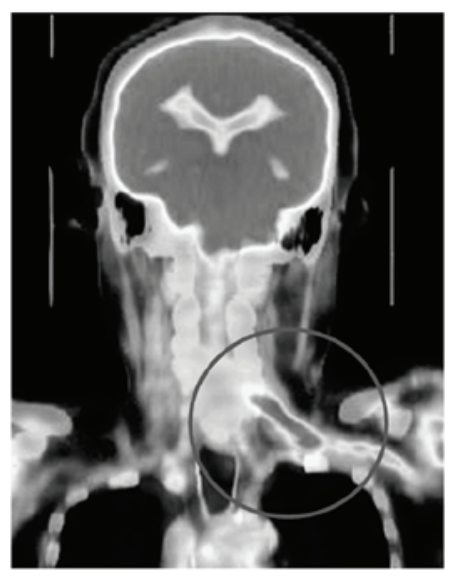

B

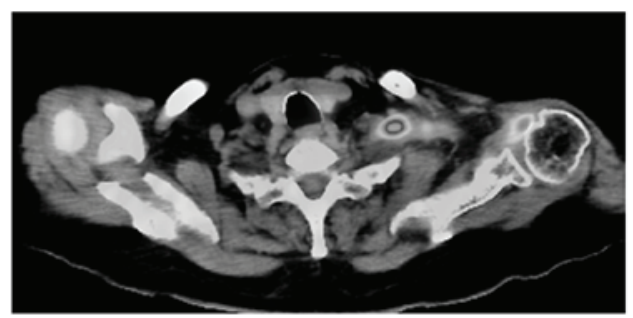

Figure 1. (A) Coronal PET-CT showing increased tracer uptake in the left brachial plexus. (B) Axial PET-CT showing a tumor arising from the cervical nerve root. PET-CT, positron emission tomography-computed tomography.

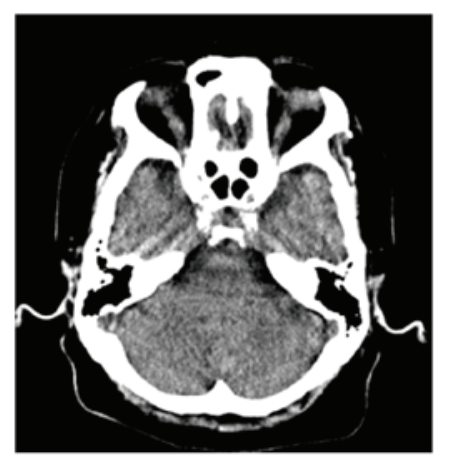

Figure 2. Plain computed tomography scan showing no significant changes in the brain.

hematoxylin and eosin. The histological findings demonstrated interweaving of atypical spindle cells with a high mitotic rate, consistent with a high-grade sarcoma. Tissues were incubated with the following antibodies: Monoclonal mouse anti-human integrase interactor 1 (INI-1; dilution, 1:2,400; \#612110; BD Biosciences, Franklin Lakes, NJ, USA), polyclonal rabbit anti-human S100A (ready for use/dilution, 1:1; \#760-2523; Ventana Medical Systems, Inc., Tucson, AZ, USA), monoclonal mouse anti-human cytokeratin (dilution, 1:800; \#M3515; Dako, Glostrup, Denmark), monoclonal mouse anti-human cluster of differentiation (CD)34 (dilution, 1:400; \#413361; Nichirei Corporation, Tokyo, Japan), monoclonal mouse anti-human p16 (dilution, 1:800; \#551153; BD Pharmingen, San Diego, CA, USA), monoclonal mouse anti-human $\alpha$-smooth muscle actin ( $\alpha$-SMA; dilution, 1:800; \#M0851; Dako) and monoclonal mouse anti-human epithelial membrane antigen (EMA; ready for use/dilution, 1:1; \#M1504;
A

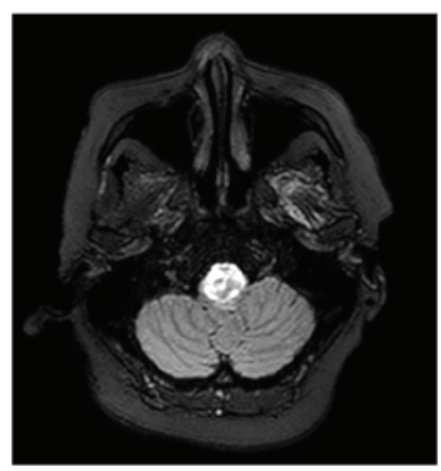

B

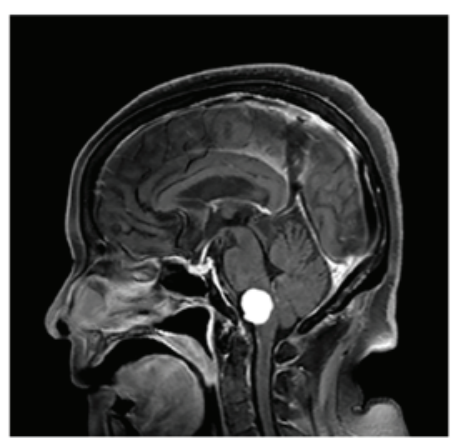

Figure 3. Gadolinium-enhanced magnetic resonance (A) axial and (B) sagittal images showing metastasis in the medulla.

Dako). Antibodies for cytokeratin, INI-1, EMA and p16 were incubated at $100^{\circ} \mathrm{C}$ for $30 \mathrm{~min}$, whereas antibodies for CD34, $\alpha$-SMA and S100A required no pretreatment. Immunohistochemical analysis demonstrated that the tumor was positive for INI-1, and negative for S100A, cytokeratin, CD34, p16, $\alpha$-SMA and EMA. As wide resection of the tumor was considered to be difficult due to the tumor location, the patient underwent carbon ion radiotherapy (70.4 Gy equivalence for a total of 16 fixed fractions for 3 weeks) at the Research Center Hospital for Charged Particle Therapy, National Institute of Radiological Sciences (Chiba, Japan). No severe acute toxicity associated with the carbon ion radiotherapy was observed.

A total of four months subsequent to administration of carbon ion radiotherapy (September 2013), the patient developed a high-grade fever $\left(40^{\circ} \mathrm{C}\right)$ and was admitted to Shingu Municipal Medical Center with suspected pyelonephritis. Laboratory data revealed an increased level of C-reactive protein $(8.05 \mathrm{mg} / \mathrm{dl}$; normal range, $<0.3 \mathrm{mg} / \mathrm{dl})$ and a normal white blood cell count $(7,400 / \mu 1$; normal range, $4,000-8,000 / \mu 1)$. A blood culture was negative for infection. Although intensive antibiotic therapy was administered (cefotiam $2 \mathrm{~g} /$ day for 12 days, followed by doripenem $1 \mathrm{~g} /$ day for 1 week), the high grade fever continued and the patient's laboratory data did not improve. CT scans of the head, chest, abdomen and pelvis revealed no source of infection. However, recurrence of the MPNST at the shoulder, an area not previously treated with carbon ion radiotherapy, was identified. No distant metastases were observed. MRI revealed tumor recurrence in the deltoid muscle. Therefore, the high-grade fever was suspected to be a neoplastic fever. Following $24 \mathrm{~h}$ treatment with naproxen (300 mg/day for 3 weeks), the fever was alleviated. The patient was subsequently referred to Mie University Hospital in October 2013 to undergo wide excision 
of the recurrent tumor. The histopathological findings revealed interweaving atypical cells with a high mitotic rate, necrosis and degeneration, consistent with a high-grade MPNST. A total of 3 weeks subsequent to surgery, the patient suddenly developed a stagger and dysarthria. Treatment with all drugs, including oxycodone and pregabalin, was discontinued due to suspected opioid overdosing; however, the symptoms did not improve. Plain CT scanning (Aquilion ONE; Toshiba Corporation, Tokyo, Japan) performed in November 2013 revealed no significant changes in the brain and chest (Fig. 2). Dysphagia emerged and left upper limb pain was alleviated the following day. MRI revealed metastasis in the medulla (Fig. 3A and B). A total of 5 days subsequent to the onset of dysarthria, the patient succumbed due to respiratory failure.

\section{Discussion}

To the best of our knowledge, no previous cases of medullary metastasis arising from a MPNST, in the absence of lung metastasis, have been reported in the literature. Although brain metastases are estimated to develop in $15-40 \%$ of cancer patients, they are considered a rare event in soft tissue sarcoma patients, with a reported prevalence of $1-6 \%$ (4). Furthermore, brain metastasis occurring in the absence of lung metastasis is considered a relatively rare event (4-6). Notably, Ogose et al (6) reported that alveolar soft part sarcoma, extraskeletal Ewing's sarcoma and rhabdomyosarcoma exhibit relatively high incidences of brain metastases. Regarding MPNSTs of the lung, only 21 cases of metastases to the brain have been reported previously (7). Of these 21 cases, the frontal lobe was the most common location for brain metastasis (7). Metastasis to the brainstem is uncommon and accounts for only 3-5\% of all brain metastases (8). Therefore, in the present case, dysphagia and stagger were initially suspected to be side effects of oxycodone treatment. The clinical presentation of brain metastasis is characterized by the acute onset of neurological symptoms $(1,7)$. The clinical symptoms of medullary metastasis may be similar to a medullary infarction, which include dysphagia, dysarthria, dysphonia, vertigo, contralateral deficits in pain and temperature sensation, and vomiting (9). The median survival time in patients exhibiting untreated metastasis to the brain is 1-2 months (10-12). Smalley et al (13) reported a median survival time of 11.7 months following resection of solitary brain metastases. Generally, brainstem metastases are not treated with conventional neurosurgery due to the risk of neurological damage $(9,10)$, and Gamma Knife surgery is typically considered for brainstem metastasis (9) Gamma knife surgery exhibits a local control rate of $83-94 \%$ with a median survival time of 7-12 months (10). In the present case, gamma knife surgery may have been an appropriate treatment for tumor control. However, the patient's general condition rapidly worsened and the patient succumbed 5 days subsequent to the initial presentation with the clinical symptoms of brain metastasis. Therefore, no treatment was administered.
Although a plain brain CT scan was performed prior to resection of the recurrent tumor, brain metastasis was not detected. MRI is a useful examination tool for the identification of brain metastasis; however, the high medical cost of MRI as a routine examination must be considered due to the rarity of such metastases. In conclusion, due to the rarity of brain metastases and the lack of information regarding treatment, follow-up treatment strategies for brain metastases arising from soft tissue sarcoma require additional investigation.

\section{Acknowledgements}

The authors would like to thank Dr R Imai from the Research Center Hospital for Charged Particle Therapy, National Institute of Radiological Sciences (Chiba, Japan) for performing carbon ion radiation.

\section{References}

1. Zhou W, Du X, Song F, Zheng H, Chen K, Zhang W and Yang J: Prognostic roles for fibroblast growth factor receptor family members in malignant peripheral nerve sheath tumor. Oncotarget: Mar 14, 2016 (Epub ahead of print).

2. Rawal A, Yin Q, Roebuck M, Sinopidis C, Kalogrianitis S, Helliwell TR and Frostick S: Atypical and malignant peripheral nerve-sheath tumors of the brachial plexus: Report of three cases and review of the literature. Microsurgery 26: 80-86, 2006.

3. Valentin T, Le Cesne A, Ray-Coquard I, Italiano A, Decanter G, Bompas E, Isambert N, Thariat J, Linassier C and Bertucci F: Management and prognosis of malignant peripheral nerve sheath tumors: The experience of the French Sarcoma Group (GSF-GETO). Eur J Cancer 56: 77-84, 2016.

4. Espat NJ, Bilsky M, Lewis JJ, Leung D and Brennan MF: Soft tissue sarcoma brain metastases. Prevalence in a cohort of 3829 patients. Cancer 94: 2706-2711, 2002.

5. Nakamura T, Matsumine A, Matsubara T, Asanuma K, Niimi R, Uchida A and Sudo A: Retrospective analysis of metastatic sarcoma patients. Oncol Lett 2: 315-318, 2011.

6. Ogose A, Morita T, Hotta T, Kobayashi H, Otsuka H, Hirata Y and Yoshida S: Brain metastases in musculoskeletal sarcomas. Jpn J Clin Oncol 29: 245-247, 1999.

7. Shweikeh F, Bukavina L, Saeed K, Sarkis R, Suneja A, Sweiss F and Drazin D: Brain metastasis in bone and soft tissue cancers: A review of incidence, interventions, and outcomes. Sarcoma 2014: 475175, 2014.

8. Lamm AF, Elaimy AL, Lamoreaux WT, Mackay AR, Fairbanks RK, Demakas JJ, Cooke BS and Lee CM: A review of the clinical outcomes for patients diagnosed with brainstem metastasis and treated with stereotactic radiosurgery. ISRN Surg 2013: 652895, 2013.

9. Lai-fung Li, Gilberto Ka-kit Leung, Ronnie Sin-lun Ho and Wai-man Lui: Recurrent natural killer cell lymphoma with central nervous system metastasis mimicking cerebellar infarction. World Neurosurg 84: 2074.e5-9, 2015.

10. Yoo TW, Park ES, Kwon do H and Kim CJ: Gamma knife radiosurgery for brainstem metastasis. J Korean Neurosurg Soc 50: 299-303, 2011

11. Lang EF and Slater J: Metastatic brain tumors: results of surgical and non-surgical treatment. Surg Clin North Am 44: 865-872, 1964.

12. Markesbery WR, Brooks WH, Gupta GD and Young AB: Treatment for patients with cerebral metastases. Arch Neurol 35: 754-756, 1978.

13. Smalley SR, Laws ER Jr, O'Fallon JR, Shaw EG and Schray MF: Resection for solitary brain metastasis. Role of adjuvant radiation and prognostic variables in 229 patients. J Neurosurg 77: 531-540, 1992. 